\title{
Unidad de Educación Continuada
}

Director: Moulin Sang.

1.- En Santo Domingo. Curso "Reglas de Refinamiento en Etiqueta y Protocolo".

Temas: 1ro. Actos sociales ( bautizos, primera comunión, open house, garden partie, cocktail, etc.). 2do. La cancillería y el protocolo, honores, el menú, etc. 3ro. Arte de preparar y servir una mesa. 4to. Los vinos. Su historia y usos. Demostración de copas y vasos para cada ocasión. 5to. Los quesos. Reglas fundamentales que todo buen gourmet debe saber y tener en cuenta.

Dirigido a: Gerentes de Personal, encargados de banquetes, personas con posiciones ejecutivas, secretarias y todos aquellos caballeros, damas y jóvenes interesados en mejorar su apariencia personal $y$ aprender las reglas fundamentales para conducirse adecuadamente según el ambiente $y$ actividad social.

Profesora: Lic. Amalia Aguilar de Brugal. Fechas: 10 de julio a 7 de agosto (grupo A). 12 horas 13 de julio a 10 de Agosto (grupo B) 12 horas.

2.- Santo Domingo Centro Regional del |B| para la Enseñanza de la Informática y departamento de Ciencias de la Computadora e Informática.

Temas: 1ro. Concepto y evolución de los sistemas operativos.

20. Estructura de los sistemas operativos.

3ro. Programas de control de gestión del sistema. Asignación de Memoria.

3 Sistemas operativos y micro-procesadores.

Dirigido a: Los profesionales de distintas disciplinas académicas interesados en la materia, con conocimiento y experiencia sobre procedimiento automáticos de datos.

Profesor: Mariano Luengo Jiménez. Fecha: 5 de Agosto a 9 de agosto (25 horas). 
3.- En Santo Domingo. Curso: "Relaciones Humanas y Oratoria". Objetivos: 1ro. Interesar al participante en la importancia de sus relaciones humanas con su familia, su trabajo y la sociedad en general 20. Diferentes ciclos de la personalidad humana. El matrimonio, su concepto, su problemática y la forma de armonizar la pareja;

Dirigido a: Personas interesadas en mejorar sus relaciones e imagen personal; como superar el miedo frente al público como su medio de trabajo.

Profesor: Dr. Francisco Brugal Muñoz y Lic. Magaly Aguilar de Brugal. Fechas: 7 de agosto a 4 de septiembre ( 12 horas) Grupo A. 10 de agosto a 7 de septiembre ( 12 horas) Grupo B.

4.- Santo domingo.- Escuela de Ingeniería Civil: Curso: "-603 Contabilidad de costo para ingenieros.. Temas 1ro. Cuentas del mayor y estructuración de los costos y gastos (Carta de cuentas; análisis de costos auditoría).

20. Contabilización de costos de materiales y servicios.

30. Diseño de un sistema de contabilizada para una empresa constructora.

4to. El uso de micro-Computadora en la contabilidad de costos. Dirigido a: Arquitectos, ingenieros civiles, estudiantes de término de una asignatura pendiente de aprobación. Profesor: Ing. Plutarco Lara.

5.- Santo Domingo: Curso Técnico Práctico: "Formulación de proyectos de inversión."

Objetivos: Dar a conocer los elementos metodológicos y técnico que les faciliten al participante presentar y evaluar objetivamente alternativas de inversión, adecuando esos conocimientos a las circunstancias económicas actuales.

Dirigido a: Funcionarios de empresas públicas y privadas, así como también a profesionales y estudiantes de término de las carreras de Administración de empresas, Economía, Contabilidad e Ingeniería.

Profesores: Miguel Sang Ben Master en Administración y en Economía y Enrique Ogando B., Ejecutivo de INDOTEC.

Fechas: 20 de julio a 28 de agosto ( 30 horas).

6.-- Santo Domingo: Biblioteca de la UNPHU. Curso: "Servicio al público en bibliotecas." Objetivos: Conocer los aspectos generales y específicos de las diferentes tareas en cada una de las seccio. nes principales del área de servicio al público, como son: Circulación, Reserva, Referencia dominicana, Hemeroteca y audiovisual.

Capacitar, entrenar y actualizar en el área de servicio al público para Libliotecas a personal auxiliar que labora en bibliotecas. 
Dirigido a: Encargados de bibliotecas, supervisores de los servicios públicos de bibliotecas barriales; auxiliares bibliotecarias. Personas y estudiantes universitarios interesados en aprender a cono. cer y usar la biblioteca como fuente de investigación.

Profesor: Lic. Altagracia Peña Cruz (Master en Ciencias Bibliotecarias). Fechas: 20 de julio a 31 de agosto.

7.- Santo Domingo: Centro Regional del IBI para la enseñan. za de la Informática (CREI) y Departamento de Ciencias de la Computadora e Informática. Cursos: "Sistemas Operativos".

Temas: 1ro. Concepto y evaluación de los sistemas operativos.

2do. Estructura de los sistemas operativos.

3o. Programas de control de gestión del sistema. Asignación de memoria.

40. Sistemas operativos y micro procesadores.

Dirigido a: Los profesionales de distinta disciplinas académicas interesadas en la materia, con conocimiento y experiencias sobre procesamiento automático de datos.

Profesor: Dr. Mariano Luengo Jiménez, Profesor del Instituto Nacional de Administración Pública y subdirector de la Empresa Nacional de Estudios, y Realizaciones de Informática Académica (ERIA).

Fechas: 5 de agosto a 9 de agosto ( 25 horas).

8.- Extensión de Santiago: Curso: "Técnicas de operación de mantenimiento de Calderas.". Objetivos: Al termino del curso los participantes habrán adquirido los conocimientos necesarios que les permitan aplicar las técnicas adecuadas para que las calderas operen con el máximo de eficiencia y seguridad.

Dirigido a: Fogoneros, operadores de máquinas, mecánicos, supervisores y jefes de plantas de proceso.

Profesor: Ing.. Héctor Urbea, Asesor Técnico-industrial. Fechas: 20 de julio a 17 de agosto ( 32 horas).

9 y 10.- Santo Domingo: Escuela de Ingeniería Civil. Cursos: "Administración de Empresas dirigidos a la construcción."

Objetivos. Ampliar las habilidades y destrezas que deberán mostrar los ingenieros civiles para satisfacer las demandas actuales y futuras del mercado de trabajo y obtener en la Escuela de Ingeniería Civil las experiencias previas para las maestrías.

Dirigido a: Ingenieros civiles y arquitectos, estudiantes de término de dichas carreras con más de una asignatura pendiente de aprobación. Fechas y profesores: 22 de julio a 22 de octubre.Ing. 604.- "Métodos de programación". Ing. Bolívar Senior. 28 de julio a 28 de octubre.- Ing 601 "Ingeniería económica". Ing. Alvaro Sánchez. 
11.- Santo Domingo. Departamento de Letras. Curso: "Metodología para la aplicación de la Literatura como documento".

Temas: 10. Elementos de la novela ((argumento, el autor, el público...)

2o. El estructuralismo y sus problemas.

3o. Las obras literarias objetivadas. El concepto de ficción.

40. La sociedad en el arte. Dirigido a: poetas, escritores, críticos literarios, periodistas, historiadores, sociólogos, estudiantes de letras Historia y Sociología, Personas interesadas: Profesora: Dra. Josefina de la Cruz, Doctora en Filosofía y Letras (Filología románicas. Fechas: 16 de julio al 9 de agosto ( 24 horas).

12. Santo Domingo. Curso: "Formulación y elaboración de proyectos de inversión."

Dirigido a: Analistas de proyectos y de préstamos economistas de proyectos y de préstamos economistas, contadores, administradores ingenieros $y$ todos aquellos profesionales que trabajan en el área de proyectos de inversión.

Profesores: Lic. Miguel Sang Ben, master en Economía. Lic. Enrique Ogando, funcionario de INDOTEC. Fechas: 29 de julio a 28 de agosto ( 30 horas).

13.- Santo Domingo: Curso: "Relación interpersonal Enfermera-Paciente". Objetivos: 10. Mejorar la relación personal enfermera-paciente sobre la base del análisis del marco emocional del paciente.

20. Favorecer la recuperación del pacientes a través de una relación personal adecuada a las necesidades del mismo.

3o. Reconocer en el paciente a una "persona" inmersa tempo. realmente una situación especial.

Dirigido a: Supervișores de enfermería, jefes de estación, enfermera graduadas, auxiliares de enfermería y estudiantes de enfermería.

Profesora: Lic. María J. San Martín Benito, de la universidad de Zaragoza(España). Fechas: 17 de julio a 14 de agosto ( 15 horas).

14 y 15.-Santo Domingo: Talleres de Verano *! "Para el desarrollo de la creatividad artística. 19 de agosto a 26 de septiembre: "Dibujo artístico". Método para facilitar la expresión artística a través de lápiz de grafito, de carbón.

Profesora: Carmen Pellerano. 19 de agosto a 26 de septiembre: "Técnicas en acuarela.".

Técnica del color y forma en acuarela. Profesora: Carmen Pellerano.

20 al 29 Santo Domingo: Departamento Lenguas Extranjeras. 
Cursos de "Lenguas Extranjeras".

1 er. Semestre.

I.- Inglés elemental.- Lic. Teresa Carrión (64 horas)

II. - Inglés Intermedio I- John Di Prieto (" ")

III.-- Inglés Intermedio II.-Ronald Block (" " ")

IV. - Inglés Avanzado I. - Kenneth Killary (" ")

V. Inglés Avanzado II.-Milagros Febles (" ")

VI.-Conversación I. - Lic. Teresa Carrión (" ")

VII.- Francés I.-Obdulia García

VIII.-Francés.-Daniel Pierre

IX Francés III.- Obdulia García

Fechas: Del 18 de Agosto al 29 de Noiviembre.

30.- Santo Domingo: Escuela de ingeniería Civil: Curso: "Técnica de Presupuestos para ingeniería: Edificaciones y Movimiento de tierra y Obras Civiles."

Temas (Parte I); Planos, especificaciones y presupuestos. Gastos directos e indirectos. Preparación del terreno (demostración replanteo...)

(Parte II) Costo de estudio en proyecto Costo de Inversión. Rendimiento de equipo. Análisis de costo del material. Análisis de costo de regado, nivelado, relleno. Dirigido a: Ingenieros Civiles, arquitectos, administradores de empresas $\mathrm{CX}$ A, profesionales encargados de asesorar oficinas de ingenieros, estudiantes de ingeniería $y$ arquitectura.

Prof: Ing. Plutarco Lara. Fechas: 27 de agosto a 29 de octubre ( 80 horas).

31.- Santo Domingo: Departamento de Letras.

Curso: "Técnica de Redacción Avanzada". Diseñado para la adquisición de conocimientos funcionales que ayuden a enfrentar cualquier tipo de redacción de carácter discursivo (cualidades de esti10, naturalidad, precisión, claridad, la estructura de párrafo, etc.)

Dirigido a: Estudiantes de letras, de Educación, periodistas, redactores de noticias, secretarias, personas interesadas en mejorar $y$ adquirir destrezas y técnicas del bien combinar palabras y sentidos en las líneas.

Prof.: José Enrique García. Fechas: 3 de septiembre a 3 de octubre.. 25 horas.

32. Santo Domingo: Curso: "Cocina china a la cantonesa y pekinesa". Platos: Carne de res con salsa de ostras, cerdo al estilo SCE, Chun con sopa de pescado, robalo en rebanada, costillas al estilo de Pekin, pollo al estilo cantonesa y otros más.

Dirigido a: Personas interesadas en conocer los platos de la cocina china. 
Fechas: 29 de agosto a 19 de septiembre.

33.- Santo Domingo: Facultad de Ciencias. Curso: "Introducción a la acuacultura". Objetivos: Transmitir a los asistentes los fundamentos básicos, teóricos y prácticos para el cultivo de organismos acuáticos en agua dulce y marinas tropicales.

Dirigido a: Biólogos, agrónomos cronistas, químicos del área alimenticia, personas relacionadas con el manejo de sistema de distribución del agua, estudio técnico financiero de proyectos de desarrollo, inversionistas. Temas: Acuacultura en ambiente lacustre, llu-

- via y marino. El elemento agua, sus componentes e incidencias ecológicas de los factores físicos, químicos y dinámicos.

- Técnicas de cultivos: peces, crustáceos, moluscos y plantas acuáticas.

- Reproducción. Alimentación.

- Metodología para la identificación, planificación y ejecución de proyectos de acuacultura: estudios de mercado, análisis técnico financiero, aspectos legales.

Profesor: Dr. Gerard Gaugé. Oceanógrafo-Biólogo. Fechas: 3 de septiembre a 26 de noviembre ( 48 horas).

34.- Santo Domingo: Departamento de Letras. Curso: "Técnicas de redacción avanzada". Diseñado para la adquisición de conocimientos funcionales $y$ destrezas que ayudan a enfrentar cualquier tipo de redacción de carácter discursivo (cualidades de estilo, naturalidad, precisión, claridad, la estructura de párrafos, etc.)

Dirigido a: Estudiantes de letras, de educación, periodistas, redactores de noticias, secretarias; personas interesadas en mejorar y adquirir destrezas y técnicas del bien combinar palabras y sonidos en las lineas.

Profesor: José Enrique García. Fechas: 3 de septiembre al 3 de octubre ( 25 horas).

35.- Santo Domingo: Centro Regional del IBI para la Enseñanza de la Informática. Curso de "Informática Agraria". Temas: -Sistema de información (proceso de desarrollo, participación del usario) modelización).

- Aplicaciones empresariales (contabilidad, inversión, rebaños,

-Aplicaciones en la administración pública (censos, estadísticas, series cronológicas, sistema de información de precios y mercados).

Dirigido a: Profesionales en el área agrícolas de instituciones públicas y privadas, así como el personal encargado de empresas agrícolas y de ganaderos. Profesor: José Luis de Miguel Arenal, de la 
Universidad Politécnica de Madrid. Fechas: 16 al 20 de septiembre ( 25 horas).

36.- Extensión de Santiago. Curso: "Introducción al control de calidad." Objetivos: Conocer los elementos fundamentales del control de calidad y su aplicación a la industria.

Dirigido a' Estudiantes de ingeniería química y personas que trabajan en el área de control de calidad o producción.

Profesores: Ing. Giovanna Divanna de Martín. Ing. Suk-Lan y Sang de Hernández. Fechas: 7 al 27 de septiembre ( 16 horas).

37.-- Santo Domingo: Curso-Taller: "De Serigrafía (Nivel básico-" Temas: -Equipo y materiales (elevación del marco raquetas, montaje de multa-)

- Colores, materiales y accesorios.

-Métodos fotográficos (directo, indirecto, y secado del traje)

-Aplicaciones de la serigrafía (inpresión del papel, metal, cristal, madera, calcomanía).

Dirigido a: Publicistas, estudiantes de arquitectura, diseño y decoración, de Artes Gráficas y diseño de bellas Artes. Profesor: Genaro Phillips. Fechas: 17 de septiembre a 24 de octubre ( 30 horas)

38.- Santo Domingo: Curso Taller: "Técnica de maquillaje y cuidados básicos de la piel".

Temas: -Análisis de la piel (tipos de piel, consejos, agua, humedad, etc.)

- Cuidados especiales para la piel.

-Cómo maquillarse (uso correcto de las cremas).

-Diferentes tipos de maquillaje (comercial, horas de trabajo, para cocktail, de fiesta formal, etc.).

Dirigido a: Demostradora de productos de belleza, propietarias de salón de belleza, estudiantes de academia de belleza, personas interesadas en aprender técnicas de maquillaje para diferentes ocasiones (oficina, cocktail, recepciones, etc.).

Profesora: Belkis Méndez, especialista en maquiliaje. Fechas 26 de septiembre a 30 de octubre ( 28 horas).

39.- Santo Domingo. Curso: "Técnica para el cuidado del paciente en estado crítico". Objetivos: -Introducir en nuestro medio el significado de "valoración del paciente" como tarea de enfermería y base para llevar a cabo un plan de cuidado razonado y preciso al paciente de alto riesgo.

- Hacer conciente al profesional de enfermería de la importancia de la elevación clínica del paciente como principal fuente de datos: estado, evolución y respuesta al tratamiento.

Dirigido a: Personal de enfermería graduado, enfermeras encargadas de estación de emergencia, asistentes de enfermería, 
auxiliares, estudiantes de medicina y enfermería: Profesora: Lic. María J. San Martín Benito. Fechas: 20 de septiembre al 25 de octubre (26 horas).

40.- Santo Domingo. Curso: "Crédito Bancario". Temas: -Importancia de las organizaciones bancarias en el desarrollo de las actividades del sector industrial y comercial. crédito.

- Requisitos para el buen funcionamiențo de un sistema de

-Análisis de las cuentas de un cliente que ganara ingresos y cuentas que generan ingresos.

- Formas de crédito bancario (Préstamos personales, transacciones especiales, líneas de crédito.

Dirigido a: Personal que labora en instituciones bancarias, estudiantes de barica, Economía, Administración, etc.

Profesor: Lic. Rafael Hued, del Banco de Reservas. Fechas: 21 de septiembre a 16 de octubre ( 76 horas).

41.- Santo Domingo. Curso: "Técnicas de la investigación científica". Objetivos: -Capacitar y entrenar a los principiantes en el planteamiento y solución de problemas de investigación.

- Dominar los procedimientos conceptuales y empíricos de la Ciencia, su método y la propia investigación científica, mediante el ordenamiento sistemático y crítico de los hechos, datos y experiencia.

- Al finalizar el curso los participantes estarán capacitados para poder llevar a cabo investigaciones con un alto grado de rigor, tanto en su vida profesional, como académica.

Dirigido a: Sociólogos, políticos, historiadores pedagogos, sicólogos y estudiantes de las carreras señaladas.

Profesor: Dr. Nelson Carreño, de la Sorbona. Fechas: 26 de septiembre a 5 de noviembre ( 24 horas).

42.- Extensión de Santiago: Curso: Sistemas de costos Estándares. Objetivos: Que los participantes conozcan a plenitud las técnicas del sistema de costo estándar, así como las ventajas que se derivan del mismo.

Dirigido a: Contadores, administradores, ingenieros industriales economistas, empresarios y todas aquellas personas vinculadas a la toma de deciciones en las empresas.

Profesor: Lic. Pedro Julio Aquino N. Fechas: 21 al 29 de septiembre (20 horas). 